\title{
Performance Evaluation of a Joint Precoding and Channel Prediction scheme for Multiuser MIMO OFDM Systems in Time Varying Wireless Channel
}

\author{
João P. Leite, Paulo H. P. de Carvalho, and Robson D. Vieira
}

\begin{abstract}
Multiple-input, multiple-output (MIMO) communications have attracted considerable attention in the last years. In particular, multiuser MIMO (MU MIMO) systems are able to offer higher link capacity of MIMO systems as long as channel state information (CSI) is available at the transmitter, so that the spatial properties of the channel can be exploited by precoding. However, the time varying nature of the channel may cause CSI to be outdated, thus degrading the overall performance of the system. Within this context, channel prediction can provide up-to-date channel CSI and reduce the performance loss. This paper presents a channel predictor based on the setmembership partial update affine projection (SM-PUAP) filtering as way to compensate the outdated CSI caused by the temporal variations of the communication channel in a multiuser MIMO OFDM (orthogonal frequency division multiplexing) system. A realistic standardized channel model is considered to evaluate the predictor and its influence on the broadcast channel of a multiuser MIMO scenario. The performance of the predictor is also compared to the well-known adaptive algorithm recursive least squares (RLS). Simulation results show that the SM-PUAP predictor has lower computational cost and superior performance when compared to the classical algorithm.
\end{abstract}

Index Terms-Adaptive filters, broadcast channel, channel prediction, multiuser multiple-input multiple-output communications, orthogonal frequency division multiplexing, time-varying channels.

\section{INTRODUCTION}

$\mathbf{O}$ RTHOGONAL frequency division multiplexing (OFDM) and systems with multiple antennas, or multiple-input, multiple-output (MIMO), are considered essential techniques for the next generation cellular systems, also referred to as $4 \mathrm{G}$ (fourth generation), considering the fact that high data rates and spectral efficiency are expected even for high mobility users [1]. Using a cyclic prefix extension of the transmitted symbol, OFDM mitigates the influence of the multipath propagation and channel selectivity, eliminating the inter-symbol interference (ISI). Multiple-input multiple-output techniques are capable of increasing the capacity of a system without the need to allocate extra bandwidth, since they provide new degrees of freedom to be explored in the spatial dimension.

MIMO techniques applied to multiuser communications (known as multiuser MIMO, or MU MIMO) have been attracting considerable attention. They are of particular interest due to the capability of offering higher link capacity of

João P. Leite and Paulo H. P. de Carvalho are with the Microwave and Wireless System Laboratory (MWSL), Department of Electric Engineering, University of Brasília, Campus Universitário Darcy Ribeiro, Asa Norte, Brasilia, DF, Brazil

Robson D. Vieira is with Nokia Technology Institute, Brasília, DF, Brazil traditional MIMO systems, provided that channel state information (CSI) is available at the transmitter [2]-[4]. By means of precoding, the spatial properties of the channel can be exploited and the multiuser interference can be mitigated [3]. This is particularly true for the downlink or broadcast channel, in which it is possible to achieve interference suppression and therefore users can receive their data symbols free from multiuser interference without any coordination among them. Two different approaches have been studied [5]: the first one, based on linear precoding and processing, is considered one of the most promising approaches due to its simplicity [6]. The second one, based on nonlinear precoding techniques, was inspired by the Dirty Paper Coding (DPC) concept and can achieve the capacity region of the MIMO broadcast channel [7], [8]. The major example of the first category of precoding techniques is the zero-forcing precoding [9], whereas the vector perturbation precoding [10] is an example of the second category. In either case, channel state information (CSI) must be available at the transmitter.

The transmitter can obtain CSI from two schemes: in frequency-division duplexing (FDD), a feedback from the receiver is used, since uplink and downlink channels are uncorrelated. In time-division duplexing (TDD), the transmitter can obtain CSI using the reciprocal property of the channel [11]. In either case, due to system constraints, such as the time-varying nature of the mobile wireless channel, scheduling algorithms and feedback delay, the CSI may be outdated, and this mismatch can severely degrade the data rate and the performance of the system. In this manner, the channel response needs to be predicted far ahead for reliable transmission and to explore full capabilities offered by multiuser MIMO systems [12].

In this paper, it is presented a multiuser MIMO OFDM channel prediction scheme based on the concept of setmembership partial update affine projection filtering (SMPUAP) as a way to compensate the outdated CSI caused by the temporal variations of the communication channel. Set-membership filtering algorithms can achieve fast rate of convergence due to the adaptive step size for each update and they present reduced update rate from its data selective nature, besides low misadjustment after convergence, since the new input data will not be used unless innovation is implied. This feature is particularly attractive if unnecessary computation must be avoided. Using the partial update strategy, the average computational complexity can be further reduced, since the algorithm benefits from the strategy and also the sparse update of the set-membership filtering.

To the best of our knowledge, although precoding [14], 
[16] and adaptive filtering have already been addressed in the literature, and in particular the use of the set-membership algorithm for channel prediction, as exposed in [13], the issues of realistic models for outdated CSI, including a timevarying channel model and feedback delay, have not yet been fully considered. Different from [13], a partial update version of the algorithm is investigated in this paper. Moreover, the effect of channel prediction on precoding techniques has been also not yet considered in the literature. Based on this exposition, the main contribution of this work is to propose the application of the SM-PUAP algorithm as an adaptive MIMO channel predictor. The proposed prediction scheme is based on a parallel single-input, single-output (SISO) channel decomposition approach [17].

In summary, the main contributions of our work are as follows:

- Modification of the prediction algorithm proposed in [13]. More precisely, we present a version based on the concept of partial update of the coefficients of the adaptive prediction filter;

- The proposal of a prediction architecture based on the decomposition of the MIMO channel into several SISO channels in order to reduce the complexity of joint MU MIMO channel prediction;

- Performance evaluation of the SM-PUAP and RLS channel predictors on a realistic channel model, the 3GPP (Third Generation Partnership Project) Spatial Channel Model;

- Performance evaluation of the joint operation of both channel precoding schemes and channel prediction algorithms.

In order to address all the issues previously mentioned, a realistic channel model and delayed feedback, in addition to realistic simulations based on the 3GPP LTE (Long Term Evolution) standard, are taken into account when analyzing the performance of two well-know precoding schemes: zeroforcing precoding (ZF) and vector perturbation precoding (VP). The performance of the proposed predictor is then compared to the RLS algorithm by means of simulations. The proposed predictor has superior performance when compared to the classical algorithm. Unlike other predictors proposed in the literature [14]-[16], it does not rely on the knowledge of channel correlation or the choice of step size.

For that matter, this paper is organized as following described: In Section II, the MIMO OFDM system model is presented. The proposed prediction scheme is presented in Section III. Section IV contains the simulation results and Section V presents the concluding remarks.

Notation We adopted the following convention: $(\cdot)^{T},(\cdot)^{H}$, $(\cdot)^{*}$ denote transpose, Hermitian transpose, and conjugate, respectively. $(\cdot)^{\dagger}$ refers to the Moore-Penrose pseudo-inverse, I represents the identity matrix, $\mathbf{0}$ represents the null vector and $\|\cdot\|$ denotes the 2-norm, given by $\|\mathbf{v}\|^{2}=\mathbf{v}^{H} \mathbf{v}$.

\section{System ModeL}

We consider the downlink of a multiuser MIMO OFDM system with $N_{D F T}$ subcarriers. A block diagram is shown in Fig. 1. There is a common base station with $N_{T}$ antennas transmitting different symbols on each subcarrier to $K \leq N_{T}$

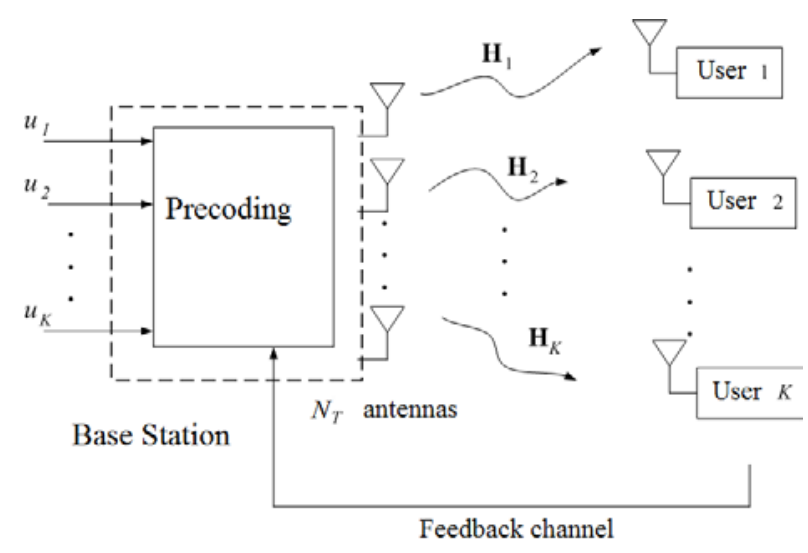

Fig. 1: MIMO broadcast channel.

users, each one with a single receive antenna. There is also a feedback channel that allows users to send channel state information to the base station, providing it with the means required to precode the information symbols and thus it can eliminate the multiuser interference.

Let $u_{k}(n, \mathscr{P})$ be the complex modulated symbol to be transmitted to the $k$-th user on the $\mathscr{P}$-th subcarrier of the $n$-th OFDM symbol. Precoding on $u_{k}(n, \mathscr{P})$ is carried out to obtain the precoded symbol $x_{k}(n, \mathscr{P})$. The signal received by the $k$-th user at the $\mathscr{P}$-th subcarrier of the $n$-th OFDM symbol, denoted by $r_{k}(n, \mathscr{P})$, can be represented as follows:

$$
\begin{aligned}
r_{k}(n, \mathscr{P}) & =\mathbf{H}_{k}(n, \mathscr{P}) \mathbf{W}_{k}(n, \mathscr{P}) u_{k}(n, \mathscr{P}) \\
& +\sum_{\substack{i=1 \\
i \neq k}}^{K} \mathbf{H}_{k}(n, \mathscr{P}) \mathbf{W}_{i}(n, \mathscr{P}) u_{i}(n, \mathscr{P})+z_{k}(n, \mathscr{P}) \\
& =\mathbf{H}(n, \mathscr{P}) \mathbf{W}(n, \mathscr{P}) \mathbf{u}(n, \mathscr{P})+z_{k}(n, \mathscr{P}) \\
& =\mathbf{H}(n, \mathscr{P}) \mathbf{x}(n, \mathscr{P})+z_{k}(n, \mathscr{P}),
\end{aligned}
$$

for $k=1, \ldots, K$ and $\mathscr{P}=1, \ldots, N_{D F T}$.

In $(1), z_{k}(n, \mathscr{P})$ is a component of additive white Gaussian noise of zero mean and variance of $\sigma_{z}^{2}$. The $1 \times N_{T}$ channel vector between the base station and the $k$-th user is given by

$$
\mathbf{H}_{k}(n, \mathscr{P})=\left[\begin{array}{lll}
H_{1,1}^{k}(n, \mathscr{P}) & \ldots & H_{1, N_{T}}^{k}(n, \mathscr{P})
\end{array}\right],
$$

the combined $K \times N_{T}$ channel matrix is

$$
\mathbf{H}(n, \mathscr{P})=\left[\begin{array}{lll}
\mathbf{H}_{1}^{T}(n, \mathscr{P}) & \ldots & \mathbf{H}_{K}^{T}(n, \mathscr{P})
\end{array}\right]^{T},
$$

and the $N_{T} \times N_{T}$ matrix

$$
\mathbf{W}(n, \mathscr{P})=\left[\begin{array}{lll}
\mathbf{W}_{1}(n, \mathscr{P}) & \ldots & \mathbf{W}_{K}(n, \mathscr{P})
\end{array}\right]
$$

represents global precoding matrix.

The complex information symbol vector is given by

$$
\mathbf{u}(n, \mathscr{P})=\left[\begin{array}{lll}
u_{1}(n, \mathscr{P}) & \ldots & u_{K}(n, \mathscr{P})
\end{array}\right]^{T},
$$

and

$$
\begin{aligned}
\mathbf{x}(n, \mathscr{P}) & =\mathbf{W}(n, \mathscr{P}) \mathbf{u}(n, \mathscr{P}) \\
& =\left[\begin{array}{lll}
\mathbf{W}_{1}(n, \mathscr{P}) & \ldots & \mathbf{W}_{K}(n, \mathscr{P})
\end{array}\right]\left[\begin{array}{c}
u_{1}(n, \mathscr{P}) \\
\vdots \\
u_{K}(n, \mathscr{P})
\end{array}\right]
\end{aligned}
$$


is the precoded symbol vector. It is important to notice that according to (1) the precoding is performed on a subcarrier basis. Moreover, we assume that the channel frequency response may vary considerably between different OFDM symbols, but it does not vary within one OFDM symbol. We also assume that an adequate guard interval is inserted in each and every OFDM symbol so ISI can be eliminated [18].

Although (6) was obtained using a linear precoding scheme (since it can be written as a product of matrices), the model is easily extended to nonlinear precoding schemes. For this matter, one has to consider that the precoded symbol vector is given by $\mathbf{x}(n, \mathscr{P})=\mathbf{f}(\mathbf{u}(n, \mathscr{P}))$, where $\mathbf{f}$ is a function (not necessarily linear) of the symbol vector $\mathbf{u}$.

Next, a few precoding schemes for the downlink of multiuser MIMO systems are reviewed. More specifically, one linear precoding technique and one nonlinear precoding technique are introduced.

\section{A. Zero-forcing Precoding}

The zero-forcing precoding, or channel inverse precoding, is the simplest form of precoding and it allows the complete suppression of the interference originated from symbols of other users [6]. At the base station, for a given instant and a given subcarrier, the information vector $\mathbf{u}(n, \mathscr{P})$ is multiplied by the pseudo-inverse of the combined channel matrix given by (3). If the number of users is less or equal to the number of transmit antennas, all co-channel interference as presented in (1) is canceled.

Mathematically, the precoding matrix is chosen as

$$
\begin{aligned}
\mathbf{W}_{Z F}(n, \mathscr{P}) & =(\mathbf{H}(n, \mathscr{P}))^{\dagger} \\
& =\mathbf{H}^{H}(n, \mathscr{P})\left(\mathbf{H}(n, \mathscr{P}) \mathbf{H}^{H}(n, \mathscr{P})\right)^{-1}
\end{aligned}
$$

Usually, $\mathbf{x}(n, \mathscr{P})$ is normalized to meet a transmission power constrained. The transmitted signal is then given by

$$
\mathbf{x}(n, \mathscr{P})=\frac{1}{\sqrt{\| \mathbf{W}_{Z F}(n, \mathscr{P}) \mathbf{u}\left(n, \mathscr{P} \|^{2}\right.}} \mathbf{W}_{Z F}(n, \mathscr{P}) \mathbf{u}(n, \mathscr{P})
$$

Despite its simplicity, the use of the inverse of the combined channel matrix leads to signal-to-noise ratio (SNR) loss and consequent capacity degradation, especially for ill-conditioned channel matrices [9].

\section{B. Vector Perturbation Precoding}

To avoid the capacity loss generated by the channel inversion precoding, the vector perturbation precoding was proposed. In this precoding technique, the symbol vector is perturbed by an integer vector as follows [10]:

$$
\mathbf{x}(n, \mathscr{P})=(\mathbf{u}(n, \mathscr{P})+\tau \ell) \mathbf{W}_{Z F}(n, \mathscr{P}) \mathbf{u}(n, \mathscr{P})
$$

where $\tau$ is a positive real number and $\ell$ is a complex vector whose real and imaginary parts are integer elements. The optimum value of the perturbation $\tau \ell$ is the one that minimizes the norm $\|\mathbf{x}(n, \mathscr{P})\|^{2}$. This is an integer-lattice least-square problem, which can be solved using sphere encoder algorithms. Nevertheless, the exact solution requires exponential complexity in $N_{T}$ [19], [20]. Unlike the zeroforcing precoding, the vector perturbation precoding is capable of achieving the capacity region of the broadcast channel.

\section{The Prediction SCHEME}

From the exposed so far, updated channel state information is required in order to perform the precoding of the information symbols. When CSI is not present at the transmitter, it is not possible to perform precoding, as can be seen from (7) and (9). Furthermore, if imperfect CSI is present at the transmitter, the interference suppression cannot be accomplished, leading to severe degradation on the performance of the system. This issue will be further addressed on Section IV, where simulation results will be shown. Due to the time-varying nature of the wireless channel, CSI might be outdated at the transmitter. To provide updated channel information, channel prediction must be considered.

\section{A. Channel Decomposition}

Before proceeding with the adaptive algorithm for channel prediction, some observations must be made. First, the prediction of the MIMO channel will be made separately for each channel coefficient. That is, the prediction of the coefficients of the matrix $\mathbf{H}_{k}(n, \mathscr{P})$ is decomposed into $N_{T}$ parallel predictors, one for each channel associated with its respective transmitting antenna [17]. Although suboptimal, this approach can greatly reduce the complexity required by joint channel prediction schemes [17], [21].

Secondly, even though the channel prediction is performed separately for each spatial channel, one must predict the channel response for each one of the subcarriers. In practical terms, it is extremely undesirable to use separated predictors for each subcarrier, since their number can be of the order of thousands [16]. This obstacle can be overcome using a time domain approach to carry on the prediction, as described in [14]. The time-varying channel coefficients $H_{1, I}^{k}(n, \mathscr{P})$, for the values of $I=1, \ldots, N_{T}$, are related to $h_{1, I}^{k}(n, l)$, the channel impulse response, by a $N_{D F T}$-point Discrete Fourier Transform (DFT):

$$
H_{1, I}^{k}(n, \mathscr{P})=\sum_{l=0}^{L} h_{1, I}^{k}(n, l) e^{-j 2 \pi \mathscr{P} l / N_{D F T}},
$$

where the variable $l$ was used to index the multiple paths of the channel impulse response, with $0 \leq l \leq L$. Let $y_{1, I}^{k}(n, l)$ denote the estimated channel response for the channel coefficient $h_{1, I}^{k}(n, l)$.

Under the assumption that the coefficients of the channel impulse response can be statistically characterized as wide sense stationary uncorrelated scattering (WSSUS), the prediction scheme consists on decomposing the channel impulse response into $L+1$ parallel single-input single-output (SISO) predictors [14]. Considering that the design of an optimal prediction filter (such as the Wiener filter) requires prior knowledge of the statistics of the channel and, on the other hand, the time varying characteristics of the channel may not always be known, an adaptive channel predictor is necessary to perform the tracking of the nonstationary channel. Using the above observations, we can write the prediction scheme as: 


$$
\hat{h}(n+p, l)=\mathbf{w}_{l}^{H}(n) \mathbf{y}(n, l), l=0, \ldots, L .
$$

It is important to remark that from (11) onwards, we have dropped the subscripts $(\cdot)_{1, I}$ and the superscript $(\cdot)^{k}$ to avoid an intricate notation. As exposed at the beginning of the section, the prediction will be carried out for each one of the spatial channels and by each one of the users, so that $(\cdot)_{1, I}$ and $(\cdot)^{k}$ are implied on the formulation.

In (11), we have $\mathbf{w}_{l}=\left[w_{0, l} \ldots w_{M-1, l}\right]^{T}$ as the vector of the predictor coefficients and the vector of regressors $\mathbf{y}(n, l)=[y(n, l) \ldots y(n-M+1, l)]^{T}$, which is the input of the prediction filter. The value of $p$ is called prediction horizon, and denotes the number of OFDM symbols the channel is predicted ahead. The value of $M$ is the number of coefficients of the filter used for channel prediction, i.e. denotes the number of samples that are being used to model the behavior of the channel as an auto-regressive (AR) process. Its choice compromises a tradeoff between accuracy and computational complexity.

The predicted channel coefficients $\hat{H}(n+p, \mathscr{P})$ are obtained from the predicted impulse response via a DFT:

$$
\hat{H}(n+p, \mathscr{P})=\sum_{l=0}^{L} \hat{h}_{l}(n+p) e^{-j 2 \pi \mathscr{P} l / N_{D F T}} .
$$

\section{B. Set-Membership Filtering}

The set-membership filtering is a framework applicable to filtering problems that are linear in parameters. Instead of specifying only one solution to the problem of designing the filter coefficients, it works by setting an upper bound $\gamma$ on the magnitude of the estimation error $\left|h(n, l)-\mathbf{w}_{l}^{H} \mathbf{y}(n-p, l)\right|$. It is worthwhile to say that this bound is a design parameter, usually varying with specific applications [22]. Its value is related to the desired rate of updating after convergence, the observation noise and the final misadjustment (after the convergence of the algorithm). Setting a value too small for $\gamma$ might lead to an empty set of feasible solutions, whereas setting a value too big might lead to inconsistent estimates. Some rules of the thumb have been suggested in the setmembership filtering literature in order to choose this upper bound properly [23], [24].

For a chosen bound, there are several estimates for the coefficients $\mathbf{w}_{l}$. The constraint set is defined as the set containing all vectors with estimation errors whose magnitude is upper-bounded by $\gamma$ at an instant $n$ :

$$
\mathscr{H}(n, l)=\left\{\mathbf{w}_{l} \in \mathbb{C}^{M \times 1}:\left|h(n, l)-\mathbf{w}_{l}^{H} \mathbf{y}(n-p, l)\right| \leq \gamma\right\}
$$

The membership set is defined as

$$
\psi(n, l)=\bigcap_{i=1}^{n} \mathscr{H}(i, l) .
$$

\section{Set-Membership Affine Projection (SM-AP)}

The membership set defined in (14) suggests that the update of the filter coefficients may be carried on using information from more than one constraint set. Using the concept of affine projection, we are going to consider the case in which the coefficient update belongs to the set formed by the last $Q$ constraint sets, $\psi^{Q}(n, l)$. This intersection is given by

$$
\begin{aligned}
\psi(n, l) & =\bigcap_{i=1}^{n-Q} \mathscr{H}(i, l) \bigcap_{j=n-Q+1}^{n} \mathscr{H}(j, l) \\
& =\psi^{n-Q}(n, l) \cap \psi^{Q}(n, l) .
\end{aligned}
$$

Whenever $\mathbf{w}_{l}(n) \notin \psi^{Q}(n, l)$, we state the following optimization criterion for updating the vector of coefficients:

$$
\begin{aligned}
& \min \left\|\mathbf{w}_{l}(n+1)-\mathbf{w}_{l}(n)\right\|^{2} \\
& \text { subject to } \mathbf{y}_{Q}(n, l)-\mathbf{Y}^{T}(n-p, l) \mathbf{w}_{l}^{*}(n+1)=\boldsymbol{\gamma}_{l}(n),
\end{aligned}
$$

where

$$
\begin{gathered}
\mathbf{y}_{Q}(n, l)=[y(n, l) y(n-1, l) \ldots y(n-Q+1, l)]^{T}, \\
\mathbf{Y}(n-p, l)=[\mathbf{y}(n-p, l) \ldots \mathbf{y}(n-p-Q+1, l)], \\
\boldsymbol{\gamma}(n, l)=\left[\gamma_{1}(n, l) \gamma_{2}(n, l) \ldots \gamma_{Q}(n, l)\right]^{T} .
\end{gathered}
$$

The term defined in (17) contains the desired outputs from the $Q$ last time instants, and (19) specifies the points belonging to the set $\psi^{Q}(n, l)$.

The minimization problem in (16) can be solved using the method of Lagrange multipliers, and it is given by [22]:

$$
\begin{aligned}
\mathbf{w}_{l}(n+1)=\mathbf{w}_{l}(n)+\mathbf{Y}(n-p, l) & {\left[\mathbf{Y}^{H}(n-p, l) \mathbf{Y}(n-p, l)\right]^{-1} \times } \\
& {\left[\mathbf{e}^{*}(n, l)-\boldsymbol{\gamma}^{*}(n, l)\right] }
\end{aligned}
$$

if $|e(n, l)|>\gamma$. If $|e(n, l)| \leq \gamma$, than there is no need for an update and

$$
\mathbf{w}_{l}(n+1)=\mathbf{w}_{l}(n) .
$$

In (20), the term $\mathbf{e}(n, l$ is the prediction error vector, expressed as

$$
\begin{aligned}
\mathbf{e}(n, l) & =\mathbf{y}_{Q}(n, l)-\mathbf{Y}^{T}(n-p, l) \mathbf{w}_{l}^{*}(n+1) \\
& =\left[\begin{array}{c}
e(n, l) \\
\varepsilon(n-1, l) \\
\vdots \\
\varepsilon(n-Q+1, l)
\end{array}\right]
\end{aligned}
$$

where $\varepsilon(n-i, l), i=2, \ldots, Q$ stands for the a posteriori error at iteration $n-i$ and $e(n, l)=y(n, l)-\mathbf{w}_{l}^{H}(n) \mathbf{y}(n-p, l)$ is an approximation of the prediction error at the instant $n$. We shall reinforce this is an approximation since we do not have access to the true channel response $h(n, l)$, but only to its estimation $y(n, l)$. In most practical cases, however, $y(n, l) \simeq h(n, l)$ for channel estimation in high SNR regime [25].

The only requirement on the parameter $\gamma_{i}(n, l)$ so far is that it must belong to the constraint set, that is, $\left|\gamma_{i}(n, l)\right| \leq \gamma$. From the choice of (19), particular solutions for (20) can be obtained. For instance, the choice $\boldsymbol{\gamma}(n, l)=\mathbf{0}$ implies that the a posteriori error are forced to zero at the last $Q$ time 
instants. Considering this case, the updating equation (20) will be identical to the conventional affine projection algorithm [23] whenever $\mathbf{w}_{l}(n) \notin \psi^{Q}(n, l)$.

Another simple version, used through the rest of the paper, can be obtained by choosing

$$
\begin{aligned}
\gamma_{i}(n, l) & =\varepsilon(n-i+1, l), i=2, \ldots, Q \\
\gamma_{1}(n, l) & =\gamma \frac{e(n, l)}{|e(n, l)|}
\end{aligned} .
$$

Geometrically, we are minimizing the Euclidean distance $\left\|\mathbf{w}_{l}(n+1)-\mathbf{w}_{l}(n)\right\|^{2}$ while keeping the a posteriori error constant at the iterations $n-i, i=2, \ldots, Q$. In this case, (20) reduces to [22],

$$
\begin{gathered}
\mathbf{w}_{l}(n+1)=\mathbf{w}_{l}(n)+\mathbf{Y}(n-p, l) \\
\quad\left(\mathbf{Y}^{H}(n-p, l) \mathbf{Y}(n-p, l)\right)^{-1} \\
\times \alpha(n, l) e^{*}(n, l) \mathbf{1} \\
\alpha(n, l)= \begin{cases}1-\frac{\gamma}{e(n, l)}, & \text { if }|e(n, l)|>\gamma \\
0, & \text { otherwise } \\
\mathbf{1}=\left[\begin{array}{ll}
1 & 0 \ldots 0
\end{array}\right]^{T}\end{cases}
\end{gathered}
$$

In order to guarantee that $\hat{h}(n+p, l)=y(n, l)$ for all values of $n=0, \ldots, p-1$, the initial state of the filter is chosen to be

$$
\mathbf{w}_{l}(n)=\left[\begin{array}{lll}
1 & 0 & \ldots
\end{array}\right]^{T}, n=0, \ldots, p-1,
$$

D. Set-Membership Partial Update Affine Projection (SMPUAP)

The partial update strategy is a very attractive way to reduce the complexity of adaptive filtering algorithms. While the recursion loop of a conventional algorithm adapts all the filter coefficients, the partial update is a framework in which $N$ out of the $M$ coefficients are updated (naturally, $N<M)$. This strategy is particularly appropriate due to the least-perturbation property of the affine projection algorithm [23]. i.e. , the algorithm tries to reduce the a posteriori error at each iteration. This behavior is further enforced by assuring that only a subset of coefficients is allowed to be updated. The idea can also be applied on the set-membership approach, reducing even further the computational costs of (21) and (24), the update equations.

The coefficients to be update are selected using an index set $\mathscr{I}(n, l)=\left\{I_{0}(n, l), \cdots, I_{N-1}(n, l)\right\}$, where the indexes are chosen from the set of all available coefficients. The optimal choice of the indexes (and consequently the coefficients to be updated) must minimize the Euclidean norm $\left\|\mathbf{w}_{l}(n+1)-\mathbf{w}_{l}(n)\right\|^{2}$. The objective function to be minimized in order to update the coefficients of the Set-Membership Partial Update Affine Projection is given as follows. A coefficient update is performed if $\mathbf{w}_{l}(n) \notin \psi^{Q}(n, l)$, as already stated. Mathematically [26],

$$
\begin{aligned}
& \min \left\|\mathbf{w}_{l}(n+1)-\mathbf{w}_{l}(n)\right\|^{2} \\
& \text { subject to: } \\
& \mathbf{y}_{Q}(n, l)-\mathbf{Y}^{T}(n-p, l) \mathbf{w}^{*}(n+1, l)=\boldsymbol{\gamma}(n, l) \\
& \tilde{\mathbf{C}}_{N}(n, l)\left[\mathbf{w}_{l}(n+1)-\mathbf{w}_{l}(n)\right]=\mathbf{0}
\end{aligned}
$$

where $\tilde{\mathbf{C}}_{N}(n, l)$ is the matrix defined in such a way that $\tilde{\mathbf{C}}_{N}(n, l) \mathbf{w}_{l}(n+1)=\tilde{\mathbf{C}}_{N}(n, l) \mathbf{w}_{l}(n)$, and only $N$ coefficients are updated. This matrix can be constructed using the relation $\tilde{\mathbf{C}}_{N}(n, l)=\mathbf{I}-\mathbf{C}_{N}(n, l)$, where $\mathbf{C}_{N}(n, l)$ is a diagonal matrix selecting the coefficients to be updated in the instant $n$. It has $N$ nonzero elements equal to one placed at the positions of the coefficients to be updated. These positions are the elements from the set $\mathscr{I}(n, l)$.

It can be shown that a recursive update equation can be found using the Lagrange multipliers [26]. The new rule is

$$
\begin{aligned}
\mathbf{w}_{l}(n+1)=\mathbf{w}_{l}(n)+ & \mathbf{C}_{N}(n, l) \mathbf{Y}(n-p, l) \mathbf{R}_{N}^{-1}(n, l) \\
& \times\left[\mathbf{e}^{*}(n, l)-\boldsymbol{\gamma}^{*}(n, l)\right]
\end{aligned}
$$

where

$$
\mathbf{R}_{N}(n, l)=\mathbf{Y}^{T}(n-p, l) \mathbf{C}_{N}(n, l) \mathbf{Y}(n-p, l) .
$$

If an update is needed, the set of indexes must be chosen in order to minimize the norm

$$
\left\|\mathbf{w}_{l}(n+1)-\mathbf{w}_{l}(n)\right\|^{2}=\left\|\mathbf{R}_{N, l}^{-1}(n)\left[\mathbf{e}_{l}^{*}(n)-\boldsymbol{\gamma}_{l}(n)\right]\right\|^{2} .
$$

In [27], it is stated that finding the index set $\mathscr{I}(n, l)$ that minimizes (31) is a binary integer programming and it cannot be solved in a computational efficient way, but some heuristics are provided. As might be expected, the choice of $\mathscr{I}(n, l)$ depends on the form of the vector $\boldsymbol{\gamma}(n, l)$. If the elements of the vector are chosen according to (23), a very simple procedure can be formulated, as described in [26]. We first rank the columns of $\mathbf{Y}^{T}(n, l)$ according to their Euclidean norm. The $N$ coefficients that will be update are those that multiply the columns of $\mathbf{Y}^{T}(n, l)$ with the $N$ larger Euclidean norms.

Intuitively, as exposed in [23], it can be observed in (29) that for a fixed value of $\left\|\mathrm{e}^{*}(n, l)-\gamma(n, l)\right\|^{2}$, the Euclidean distance between consecutive coefficient vectors is minimized if $\mathbf{R}_{N}(n, l)$ is maximized. It seems reasonable that a natural choice for the coefficients to be updated in a given instant are those that will be multiplied by the elements of $\mathbf{Y}^{T}(n, l)$ with larger norms. Once the coefficients to be updated are determined, (24) is used.

\section{E. Computational Complexity}

The computational complexity of the prediction step described in (12) is $\mathscr{O}\left(N_{T} N_{D F T} \log _{2} N_{D F T}+N_{T} M L\right)$, regardless of the adaptive algorithm. In terms of complexity, the difference between the predictors relies on the cost of updating the predictor coefficients. From the results presented in [14], one is able to infer that the update of RLS has a computational complexity of $\mathscr{O}\left(N_{T} M^{2} L\right)$. Considering (24), the SM-PUAP has computation complexity at most equal to $\mathscr{O}\left(N_{T} Q^{2} M L\right)$, the complexity of the classical affine projection algorithm [23]. Generally, the number of reuses $Q \ll M$, so the affine projection algorithm is computationally less complex than RLS.

\section{Simulation Results}

In order to evaluate the performance of the proposed channel predictor, several simulations were performed. We considered a structure similar to the downlink of 3GPP LTE standard. Next it is presented the fundamental characteristics of the system, the simulation scenario and the results. 


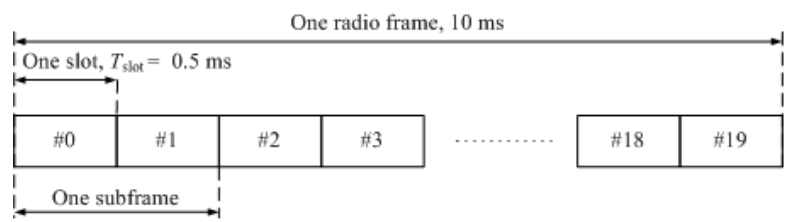

Fig. 2: Frame structure used for simulation.

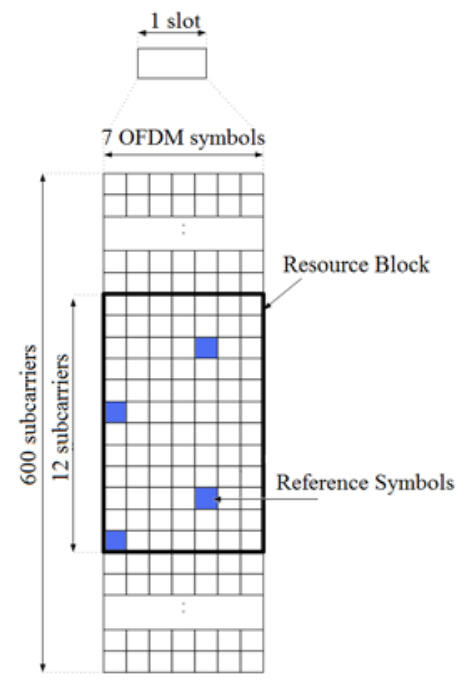

Fig. 3: Subframe structure and pilot pattern.

\section{A. Frame Structure}

For simulation purposes, a $10 \mathrm{MHz}$ bandwidth was considered. The frame structure is similar to the one found in 3GPP LTE standard. A frame is $10 \mathrm{~ms}$ long and contains 10 subframes of $1 \mathrm{~ms}$, as shown in Fig. 2. Each subframe is divided into 2 slots, each of which carrying 7 OFDM symbols. The subcarrier spacing is fixed at $15 \mathrm{kHz}$. The cyclic prefix (CP) length was chosen to be $4.6 \mu$ s [28].

As one can be observe in Fig. 3, a physical resource block is defined as 7 OFDM symbols in the time domain and 12 subcarriers in the frequency domain. LTE technology uses an optimized reference symbol allocation for channel estimation [28], [29]. In the LTE the base station can have up to 4 antennas and when two or more transmitter antennas are applied, the reference symbols are transmitted such that they are orthogonal in space. This orthogonality is obtained by letting all other antennas be silent in the resource element in which one antenna transmits a reference symbol [28]. These aspects were taken into account in order to perform the simulations.

\section{B. Mobile Wireless Channel Model}

In order to perform realistic simulations a channel with time-varying behavior has been investigated. The chosen channel model is the Spatial Channel Model Extended (SCME), which generates channel coefficients based on 3GPP channel model specifications [30]. This model considers a number of clusters of scatterers, where each cluster corresponds to a path (same relative delay) and each path consists of a given number of subpaths. The WINNER's SCME [31] was a set of
TABLE I: SCME Channel Parameters

\begin{tabular}{ll}
\hline Parameter & Value \\
\hline Carrier frequency & $2 \mathrm{GHz}$ \\
User speed & $5 \mathrm{~m} / \mathrm{s}, 30 \mathrm{~m} / \mathrm{s}$ \\
Number of antennas at base station & 4 \\
Number of antennas at user terminal & 1 \\
Scenario & Urban Micro \\
Number of paths & 19 \\
\hline
\end{tabular}

MATLAB ${ }^{\circledR}$ scripts used to provide samples of a multipath channel according to the 3GPP Spatial Channel Model. The parameter settings are shown in Table I and were used in all the simulations, unless indicated otherwise.

\section{Simulation Results and Discussion}

The channel estimation was performed using the Minimum Mean Square Error (MMSE) Estimator described in [32]. The SNR was set in $20 \mathrm{~dB}$ and the upper bound $\gamma$ for the construction of the constraint sets related to the SM-PUAP was chosen to be $\gamma=2 \sqrt{\sigma_{z}^{2}}$. This value coincides with those suggested in the literature [24]. The simulations consisted of 1000 independent runs, and the average performance is computed. In each run, 1000 OFDM symbols were transmitted.

Before proceeding with the presentation of the simulation results, a few considerations must be made. First, as presented in [13], the normalized least mean squares (NLMS) filtering exhibited the worst performance among the adaptive algorithms, despite its simplicity. For this reason we have excluded to consider the NLMS in the simulations.

Secondly, although algorithms such as QR-RLS [23] exhibit better numerical stability and present a low computational cost when compared to the conventional RLS, they present the same performance of the conventional RLS in terms of convergence rate and misadjustment in infinity precision arithmetic. Moreover, algorithms such as FT-RLS (Fast Transversal RLS) have lower computational complexity than RLS, but their performance is upper bounded by RLS. For this reason, we have considered only the performance of the classical RLS instead of its reduced complexity counterparts, following the methodology presented in [14].

The RLS algorithm was set with $M=10$ coefficients and a forgetting factor $\lambda=0.99$. The SM-PUAP was set with $M=30$ coefficients, $Q=3$ reusable sets and $N=10$ coefficients to be updated in every iteration. The number of sets used for update is a tradeoff between final misadjustment and computational complexity, as stated in Section III-E. Several simulations were performed using different values of $Q$ and it was observed that the prediction mean square error (MSE) increased about $1 \mathrm{~dB}$ from $Q=1$ to $Q=5$, so the choice $Q=3$ seemed to be a reasonable value.

For the sake of comparison, two different scenarios were considered: one in which the user speed is $5 \mathrm{~m} / \mathrm{s}$ (low mobility), and other where the user speed is $30 \mathrm{~m} / \mathrm{s}$ (high mobility). Two prediction horizons will be considered: 1 OFDM symbol and 14 OFDM symbols. The choice of 14 symbols is justifiable since the transmission time interval (TTI) of LTE is $1 \mathrm{~ms}$, or 14 OFDM symbols, as presented in Section IV-A. 


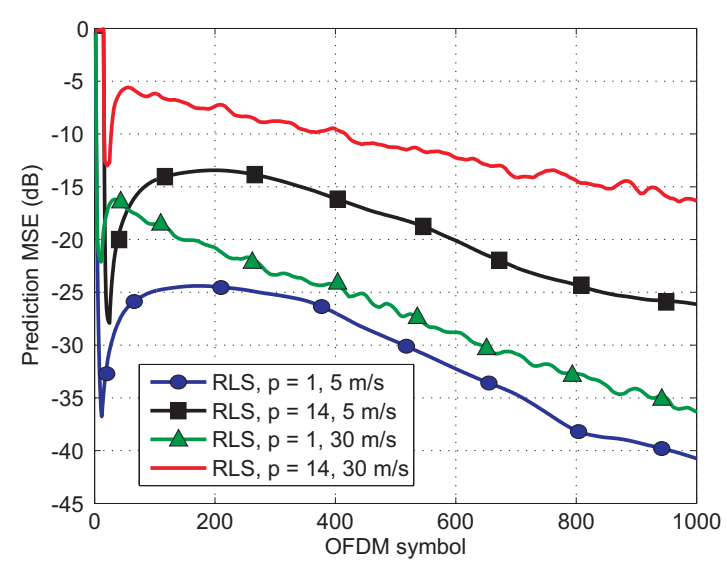

Fig. 4: Convergence behavior of the RLS adaptive predictor for a prediction horizon of 1 and 14 OFDM symbols.

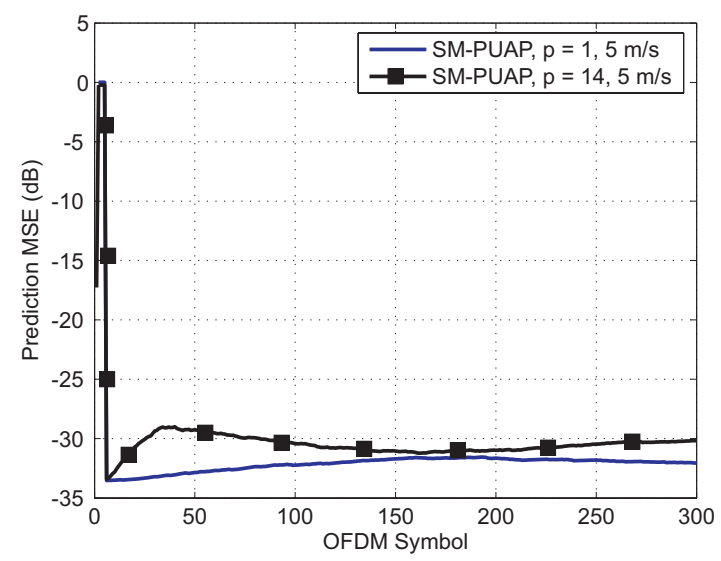

Fig. 5: Convergence behavior of the SM-PUAP adaptive predictor for a prediction horizon of 1 and 14 OFDM symbols and user speed of $5 \mathrm{~m} / \mathrm{s}$.

Fig. 4 shows the convergence of the RLS predictor for the prediction horizons of 1 OFDM symbol and also for 14 OFDM symbols. Fig. 5 illustrates the behavior of the SMPUAP for the low mobility scenario, while Fig. 6 shows its convergence for the high mobility scenario. As expected, a larger prediction horizon in a high mobility scenario slows down the convergence. The convergence of SM-PUAP was faster than the one of the RLS, although the MSE of the latter is slightly lower in the low mobility scenario. Nevertheless, in the high mobility scenario, the SM-PUAP exhibits better performance than the RLS predictor with less computational effort. One is able to infer that this occurs due to the superiority of SM-PUAP in respect to the tracking in this scenario, and such result is further reinforced the results shown in [13]. In low mobility scenarios, the RLS exhibits a slightly better performance.

To address the matter of computational effort, Table II compares the number of updates required by the channel predictors. It shows that at every iteration, the RLS algorithm demands coefficient updates. On the other hand, the SM-PUAP algorithm had its coefficients updated only in $38 \%$ of the 1000 iterations. Once the SM-PUAP updates do not occur frequently, its overall complexity is much less than that of

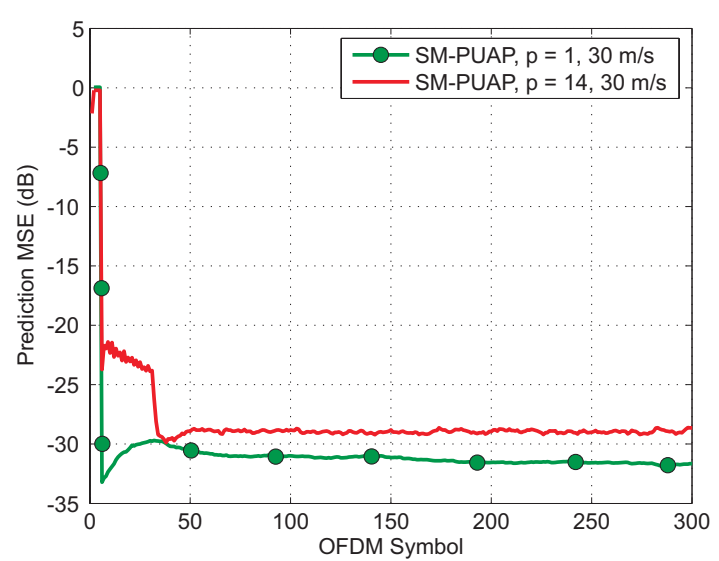

Fig. 6: Convergence behavior of the SM-PUAP adaptive predictor for a prediction horizon of 1 and 14 OFDM symbols and user speed of $30 \mathrm{~m} / \mathrm{s}$.

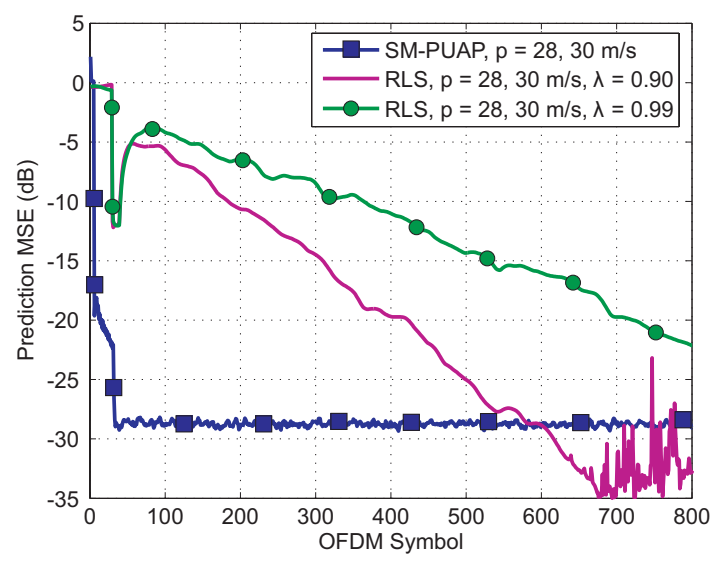

Fig. 7: Convergence behavior of the SM-PUAP and RLS adaptive predictors for a prediction horizon of $28 \mathrm{OFDM}$ symbols and user speed of $30 \mathrm{~m} / \mathrm{s}$.

the RLS counterpart. The reduced complexity is extremely desired and important in many applications, especially when power savings are mandatory, such as the power management on mobile stations. Since the SM-PUAP does not perform any unnecessary filter update, power can be saved. If properly exploited, the partial update behavior can also be used to reduce the signaling required to transmit the channel state information to the transmitter, since the radio resources of the feedback channel (if presented) are very scarce.

It is interesting to point out that the final MSE obtained using the strategy of partial update of the set-membership affine projection algorithm led to a larger prediction horizon than the one reported in [13]. One possible explanation for this behavior is that the least-perturbation property of affine

TABLE II: Comparison between the adaptive algorithms

\begin{tabular}{ll}
\hline Algorithm & Number of updates \\
\hline RLS & 1000 \\
SM-PUAP $(p=1,5 \mathrm{~m} / \mathrm{s})$ & 20 \\
SM-PUAP $(p=1,30 \mathrm{~m} / \mathrm{s})$ & 52 \\
SM-PUAP $(p=14,5 \mathrm{~m} / \mathrm{s})$ & 87 \\
SM-PUAP $(p=14,30 \mathrm{~m} / \mathrm{s})$ & 380 \\
\hline
\end{tabular}




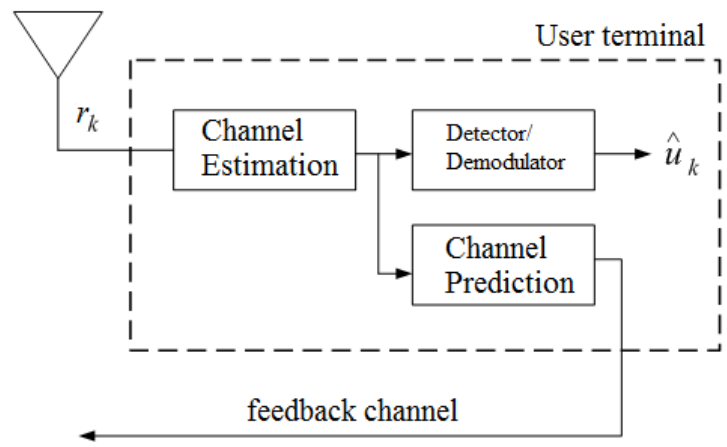

Fig. 8: Block diagram of the user terminal.

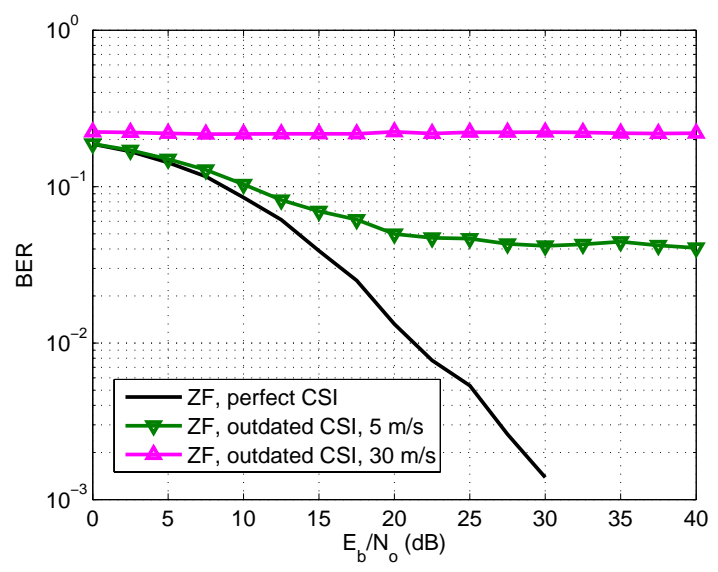

Fig. 9: BER performance of zero-forcing precoder under 16QAM uncoded modulation and $K=4$ users.

projection algorithms is further reinforced by the data-selective coefficient updating (partial update) strategy. This is a very interesting result not only due to the larger prediction horizon that can be obtained, but also the reduced computational effort when compared to the RLS algorithm (or even the SM-AP [13]). As a remark, if one tries to increase the value of the forgetting factor $\lambda$ of the RLS algorithm as a way to increase its convergence speed this will lead to a higher MSE, as well as a possible instability of the algorithm. On the other hand, decreasing the value of $\lambda$ might be helpful for larger prediction horizons at the cost of convergence speed. This sensitivity to the choice of the parameters of the algorithm is not observed for the SM-PUAP filtering. These results are summarized in Fig. 7, where it is shown the learning curves of both algorithms for a prediction horizon of 28 OFDM symbols, or 2 TTI's.

Finally, we assess the bit error rate (BER) performance of the overall MIMO OFDM system under multiuser precoding. Fig. 8 illustrates the block diagram of the process at each user. The CSI at the transmitter is implemented by an explicit closed-loop feedback. It is assumed an ideal unquantized feedback channel, and the results focus solely on the effects of channel prediction, since any other form of feedback (such as a quantized or an AWGN feedback channel) will decrease the accuracy of the prediction. The user terminal predicts the channel response and feeds the prediction via the feedback channel. In these simulations, the prediction horizon corresponds to 14 OFDM symbols, the TTI of the 3GPP LTE

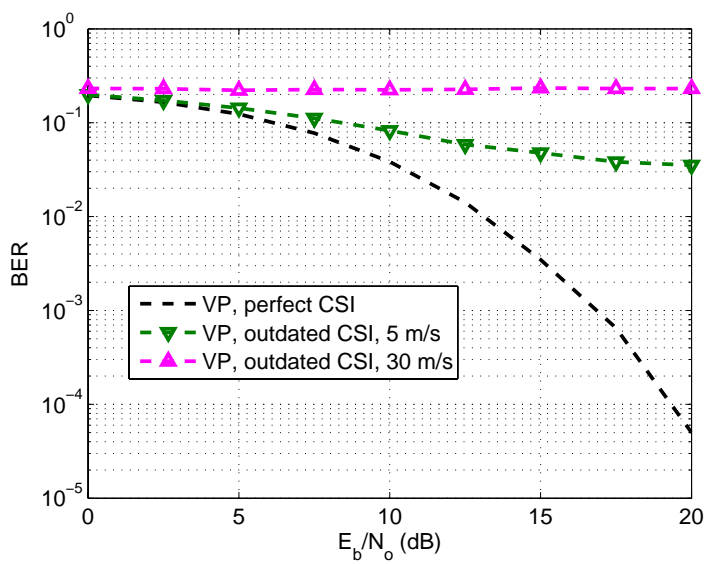

Fig. 10: BER performance of vector perturbation precoder under 16QAM uncoded modulation and $K=4$ users.

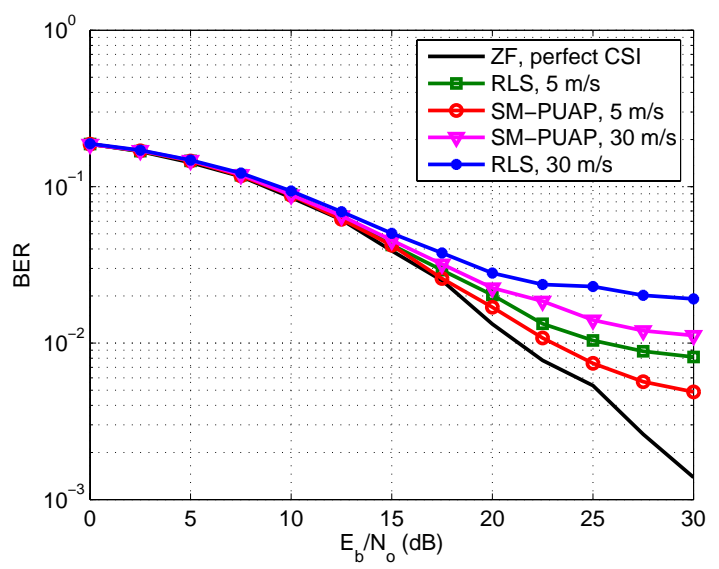

Fig. 11: Performance of the RLS and SM-PUAP channel predictors using zero-forcing precoding and 16QAM modulation.

standard.

Fig. 9 and Fig. 10 illustrate the behavior of the precoding schemes under perfect CSI and also their behavior when outdated CSI is used for the purposes of precoding. More specifically, they show the performance of zero-forcing precoding and vector perturbation precoding if no predicted channel information was available at the transmitter, therefore existing a mismatch of $1 \mathrm{~ms}$ between the information that is used for precoding and the actual channel response. As one can notice, even for a terminal of low mobility, the CSI mismatch can severely degraded the performance of the precoding schemes. The degradation is even worse for high mobility terminals due to the coherence time of the channel. Using the theory exposed in [33], the coherence time of the channel is estimated as $2.2 \mathrm{~ms}$ ( 28 OFDM symbols) for a user speed of $5 \mathrm{~m} / \mathrm{s}$, and $0.32 \mathrm{~ms}$ (4 OFDM symbols) for a speed of $30 \mathrm{~m} / \mathrm{s}$. Clearly, the higher the mobility, the faster the CSI becomes outdated.

Fig. 11 shows the performance of $\mathrm{ZF}$ precoding when channel prediction is considered, whereas Fig. 12 shows the performance of VP precoding. As a general observation, the $\mathrm{VP}$ precoding is more robust to prediction errors than the $\mathrm{ZF}$ precoding technique, and for values of $\frac{E_{b}}{N_{o}}$ below $10 \mathrm{~dB}$, the 


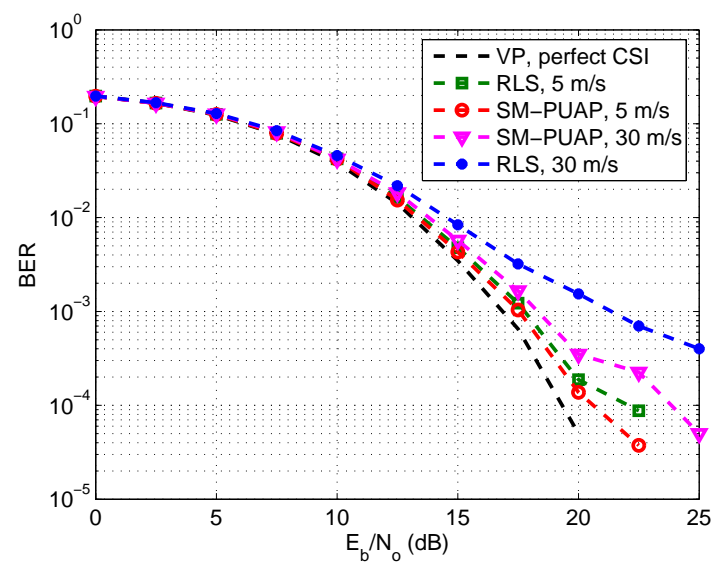

Fig. 12: Performance of the RLS and SM-PUAP channel predictors using vector perturbation precoding and 16QAM modulation.

performance of the predictors does not differ much, since it is limited by the quality of the channel estimation. Whereas all algorithms performed similarly in scenarios where the user speed is $5 \mathrm{~m} / \mathrm{s}$, the differences between them are most pronounced at higher speed. Clearly, the SM-PUAP algorithm outperforms the RLS algorithm in all cases, especially for the high mobility scenario.

As a general observation, the VP precoding is more robust to prediction errors than the $\mathrm{ZF}$ precoding technique, and for values of $\frac{E_{b}}{N_{o}}$ below $10 \mathrm{~dB}$, the performance of the prediction algorithms does not differ much since it is limited by the quality of the channel estimation. And most important, it is clear that channel prediction is capable of recovering part of the performance loss of the precoding schemes due to the outdated CSI.

\section{CONCLUSION}

In this paper, an adaptive channel predictor based on the set-membership partial update affine projection algorithm was presented for multiuser MIMO OFDM communications over time-varying channels. More specifically, the downlink or broadcast channel under two different precoding schemes was considered.

Multiuser MIMO systems are capable of offering higher link capacity of MIMO systems and interference cancellation through precoding, as long as channel state information is available at the transmitter. However, the time-varying nature of the mobile radio channel and the predicted channel state information are usually not taken into account when analyzing the performance of precoding schemes. The practical importance of channel prediction relies on providing up-to-date CSI by compensating for delay or the time varying nature of the mobile radio channel.

The performance of the developed predictor was compared to the predictor based on the RLS algorithm through simulations using a realistic channel model, the 3GPP SCME. The SM-PUAP algorithm exhibited a lower steady state MSE and a faster convergence than the RLS algorithm in high mobility scenarios. The RLS presented a slight lower MSE in low mobility scenarios and shorter prediction horizons. In spite of this fact, the SM-PUAP predictor presented lower computational costs when compared to the RLS predictor.

Finally, the impact of both predictors was considered in the bit error rate performance of two well-known precoding schemes for the downlink of multiuser MIMO systems: zeroforcing precoding and vector perturbation precoding. For this matter, a frame structure similar to the one found in the 3GPP LTE standard was considered, as well as an ideal feedback channel to provide CSI to the transmitter (base station). First, it was shown that even for low mobility scenarios, the performance of both precoders was severely degraded when outdated CSI was used to carry out the precoding, presenting the scope for the use of channel prediction. The simulation results also showed that the predictor based on SM-PUAP outperformed the one based on the RLS adaptive algorithm, especially in scenarios of larger prediction horizons and higher mobility. Moreover, it presented less computational effort than the predictor based on RLS adaptive algorithm and it was able to compensate the outdated CSI that would be available at the transmitter if no channel prediction was considered.

\section{REFERENCES}

[1] ITU-R Draft New Recommendation, "Vision, framework and overall objectives of the future development of IMT-2000 and systems beyond IMT-2000".

[2] G. Caire, S. Shamai, "On the achievable throughput of a multiantenna Gaussian broadcast channel," IEEE Trans. Inf. Theory, vol. 43, pp. 1691-1706, July 2003.

[3] Q. H. Spencer, C. B. Peel, A. Lee Swindlehurst, M. Haardt, "An introduction to the multi-user MIMO downlink," IEEE Communications Magazine, vol. 42, pp. 60-67, Oct. 2004.

[4] P. Viswanath, D. N. C. Tse, "Sum capacity of the vector Gaussian broadcast channel and uplink-downlink duality," IEEE Trans. Info. Theory, vol. 49, pp. 1912-1921, Aug. 2003.

[5] L. Choi, R. D. Murch, "Transmit preprocessing technique for multiuser MIMO systems using a decomposition approach,' IEEE Trans. Wireless Commun., vol. 3, no. 1, pp. 20-24, Jan. 2004.

[6] T. Haustein, C. von Helmolt, E. Jorswieck, V. Jungnickel, V. Pohl, "Performance of MIMO systems with channel inversion," IEEE 55th Vehicular Technology Conference, vol. 1, pp. 35-39, May 2002.

[7] C. B. Peel, "On dirty-paper coding," IEEE Signal Proc. Mag., pp. 112-113, May 2003.

[8] S. Vishwanath, N. Jindal, A. Goldsmith, "Duality, achievable rates, and sum-rate capacity of Gaussian MIMO broadcast channels," IEEE Trans. Inf. Theory, vol. 49, no. 10, pp. 2658-2668, Oct. 2003.

[9] C. B. Peel, B. M. Hochwald, A. L. Swindlehurst, "A vector perturbation technique for near-capacity multiantenna multiuser communication Part I: Channel inversion and regularization," IEEE Trans. Commun., vol. 53, no. 1, pp. 195-202, Jan. 2005.

[10] C. B. Peel, B. M. Hochwald, A. L. Swindlehurst, "A vector perturbation technique for near-capacity multiantenna multiuser communication Part II: Perturbation,” IEEE Trans. Commun., vol. 53, no.3, pp. 537-544, March 2005.

[11] C. Min, N. Chang, J. Cha, J. Kang, "MIMO-OFDM Downlink Channel Prediction for IEEE 802.16e Systems Using Kalman Filter," Wireless Communications and Network Conference, pp. 942-946, Mar. 2007.

[12] A. Duel-Hallen, "Fading Channel Prediction for Mobile Adaptive Transmission Systems," Proc. IEEE, pp. 2299-2313, Dec. 2007.

[13] J. P. Leite, P. H. P. de Carvalho, and R. D. Vieira, "OFDM channel prediction using set-membership affine projection algorithm in timevarying wireless channel," IEEE 10th Workshop on Signal Processing Advances in Wireless Communications 2009 (SPAWC 09), Perugia, Italy, pp. 26-30, June, 2009.

[14] D. Schafhuber, G. Matz, "MMSE and Adaptive Prediction of TimeVarying Channels for OFDM Systems," IEEE Trans. Wireless Commun., vol. 4, no. 3, pp. 593-602, Mar. 2005.

[15] J. Wen, C. Chang, G. Lee, C. Huang, "OFDM Channel Prediction Using Fuzzy Update LMS Algorithm in Time-Variant Mobile Channel," 64th IEEE Vehicular Technology Conference, pp. 1-5, 2006. 
[16] A. Duel-Hallen, H. Hallen, Tung-Sheng Yang, "Long Range Prediction, and Reduced Feedback for Mobile Radio Adaptive OFDM Systems," IEEE Trans. Wireless Commun., vol. 4, no. 10, pp. 2723-2733, Oct. 2006.

[17] T. R. Ramya, S. Bhashyam, "On Using Channel Prediction in Adaptive Beamforming Systems," 2nd International Conference on Communication Systems Software and Middleware, 2007. COMSWARE 2007, pp. 1-6, January 2007.

[18] H. Schulze, C. Lueders, Theory and Applications of OFDM and CDMA: Wideband Wireless Communications, Wiley, West Sussex, 2005.

[19] B. Hassibi, H. Vikalo, "On the expected complexity of sphere decoding," in Conf. Record on the 35th Asilomar Conf. on Signals, Systems and Computers, vol. 2, pp. 1051-1055, Nov. 2001.

[20] C. Windpassinger, R. F. H. Fischer, J. B. Huber, "Lattice-reduction aided broadcast precoding," IEEE Trans. Commun., vol. 52, no. 12 pp. 2057-2060, Dec. 2004.

[21] T. Svantesson, A. L. Swindlehurst, "A Performance Bound for Prediction of MIMO Channels," IEEE Transactions on Signal Processing, v. 54, n. 2, pp. 520-529, February 2006.

[22] S. Werner, P. S. R. Diniz, "Set-Membership Affine Projection Algorithm,” IEEE Signal Processing Letters, vol. 8, no. 8, pp. 231-235, Aug. 2001.

[23] P. S. R. Diniz, Adaptive Filtering: Algorithms and Practical Implementation, Kluwer, Dordrecht, 2002.

[24] J. F. Galdino, J. A. Apolinário Jr, M. L. R. de Campos, "A SetMembership NLMS Algorithm with Time-Varying Error Bound," Proc. IEEE Intern. Symmposium on Circuits and Systems, Island of Kos, Greece, pp. 277-280, May, 2006.

[25] A. van den Bos, Parameter Estimation for Scientists and Engineers, Wiley, New York, 2007.

[26] P. S. R. Diniz, G. O. Pinto, A. Hjorungnes, "Data Selective PartialUpdate Affine Projection Algorithm," IEEE International Conference on Acoustics, Speech and Signal Processing, 2008, pp. 3833-3836, April. 2008.

[27] K. Dogancay, O. Tanrikulu, "Selective Partial-Update NLMS and Affine Projection Algorithms for Acoustic Echo Cancellation," Proc. IEEE International Conference on Acoustics, Speech, and Signal Processing, Istanbul, pp. 448-451, Jun. 2000.

[28] 3GPP, "3GPP TR 36.211 V8.5.0 - Physical Channels and Modulation (Release 8)," Technical report, 3GPP, Dec. 2008

[29] C. Ball, T. Hindelang, I. Kambourov, S. Eder, "Spectral Efficiency Assessment and Radio Performance Comparison Between LTE and WiMAX," IEEE 19th International Symposiums on. Personal, Indoor and Mobile Radio Communications (PIMRC 2008), 2008, pp. 1-6.

[30] 3GPP, "3GPP TR 25.996 V8.5.0 - Spatial Channel Model for Multiple Input Multiple Output (MIMO) Simulations (Release 6),” Technica report, 3GPP, Sep. 2003.

[31] Jari Salo, Giovanni Del Galdo, Jussi Salmi, Pekka Kyoesti, Marko Milojevic, Daniela Laselva, Christian Schneider, "MATLAB implementation of the 3GPP Spatial Channel Model (3GPP TR 25.996)," On-line, January 2005. http://www.tkk.fi/Units/Radio/scm/.

[32] L. Somasegaran, "Channel Estimation and Prediction in UMTS LTE," Master thesis, Aalborg University, June, 2007.

[33] B. H Fleuty, "An Uncertainty Relation for WSS Processes and Its Application to WSSUS Systems," IEEE Transactions on Wireless Communications, v. 44, n. 12, pp. 1632-1634, Dec. 1996.

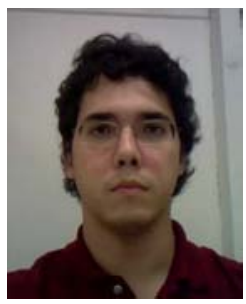

João Paulo Leite received his B.S. degree in Communication Networks Engineering in 2007 and his M.Sc. degree in Electrical Engineering in 2009, both from the University of Brasilia, Brazil. He is currently working toward a Ph.D. degree in Electrical Engineering at the same institute. His primary research interests include adaptive digital signal processing, simulation of communication systems, telecommunication software design and implementation and measurement of mobile radio channel.

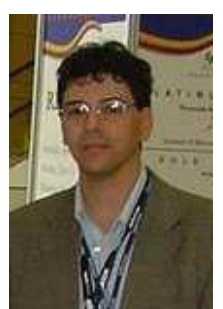

Paulo Henrique Portela de Carvalho received his B.S. in Electrical Engineering from University of Brasilia, Brazil, in 1988, his D.E.A. en Communications Optiques et Microondes from University of Limoges, France, in 1989, and his Ph.D. at Communications Optiques et Microondes from University of Limoges, France, in 1993. Currently is Professor of Electrical Engineering at University of Brasilia. He has experience in the area of Electronic Engineering, with emphasis on telecommunication systems and microwave circuit simulation.

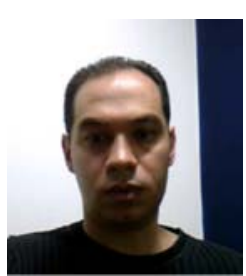

Robson Domingos Vieira received the B.S degree in Electrical Engineering from the Federal University of Goiás, Brazil, in 1999, M.Sc. and the Ph.D. degree in Electrical Engineering from the Catholic University of Rio de Janeiro, Brazil, in 2001 and 2005 respectively. From March 1999 to March 2005, he worked as a researcher assistant at Centro de Estudos em Telecomunicações (CETUC), Rio de Janeiro, Brazil. Since 2005 he has worked at Nokia Technology Institute as a Telecommunication Specialist Researcher, where he is mainly working with White Space and Cognitive radio concepts and he is giving some support to GERAN standardization in the topic M2M. In the past he worked with WiMAX/LTE simulations and gave support for standardization activities on IEEE $802.16 \mathrm{~m}$. He is also Associated Researcher of University of Brasilia. His research interests include measurement and modeling of mobile radio channel, MIMO communication systems and cognitive radio. 